Marianne Leuzinger-Bohleber (Hrsg.) · Psychoanalytische Kurztherapien 
Marianne Leuzinger-Bohleber (Hrsg.)

\section{Psychoanalytische Kurztherapien}

Zur Psychoanalyse in Institutionen 
(๑) 1985 Westdeutscher Verlag GmbH, Opladen Umschlaggestaltung: Horst Dieter Bürkle, Darmstadt

Alle Rechte vorbehalten. Auch die fotomechanische Vervielfältigung des Werkes (Fotokopie, Mikrokopie) oder von Teilen daraus bedarf der vorherigen Zustimmung des Verlages.

ISBN-13: 978-3-531-11740-9 e-ISBN-13: 978-3-322-83956-5

DOI: $10.1007 / 978-3-322-83956-5$ 
Inhaltsverzeichnis

Vorwort

I. Psychoanalytische Kurztherapie: Zur angewandten Psychoanalyse in Institutionen Eine Einführung M. Leuzinger-Bobleber

1. Psychoanalyse und Institution $\ldots \ldots \ldots \ldots \ldots \ldots \ldots \ldots \ldots$

1.1 Psychoanalytische Kurztherapien im Rahmen von Institutionen . . . 3

1.2 Psychoanalytische Kurztherapien und ihre Beziehung zu psychoanalytischen Ausbildungsinstitutionen $\ldots \ldots \ldots \ldots \ldots \ldots, 8$

2. Zum Begriff: Psychoanalytische Kurztherapie $\ldots \ldots \ldots \ldots \ldots \ldots$

3. Zur Geschichte der Psychoanalytischen Kurztherapie ......... 11

Exkurs von $A$. Benz: Eindrücke vom Internationalen Symposium zur Psychoanalytischen Kurztherapie in Alicante, $1983 \ldots \ldots \ldots \ldots \ldots 17$

4. Die Kontroverse zwischen Lang-Zeit-Therapie und Kurz-Zeit-Therapie . . 19

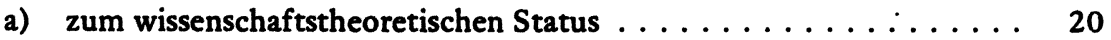

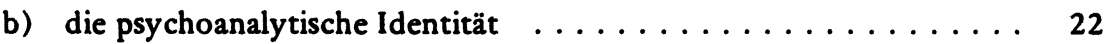

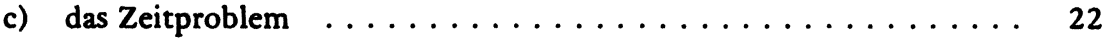

5. Kurztherapeutische Behandlungskonzepte und spezifische technische

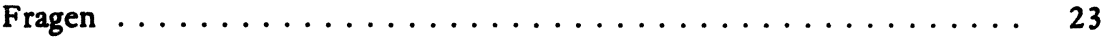

5.1 Spezifische technische Fragen $\ldots \ldots \ldots \ldots \ldots \ldots \ldots 23$

d) die Aktivität des Psychoanalytikers $\ldots \ldots \ldots \ldots \ldots \ldots \ldots 24$

e) die Neutralität des Analytikers $\ldots \ldots \ldots \ldots \ldots \ldots \ldots \ldots 24$

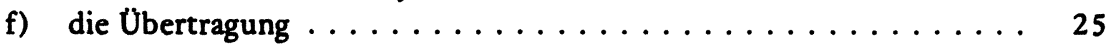

g) die Bedeutung des Hier und Jetzt $\ldots \ldots \ldots \ldots \ldots \ldots \ldots 25$

h) der Fokus und das Ziel der PKT $\ldots \ldots \ldots \ldots \ldots \ldots \ldots \ldots 26$

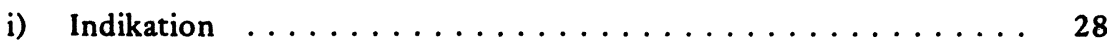

5.2 Einige kurztherapeutische Behandlungskonzepte . . . . . . 29

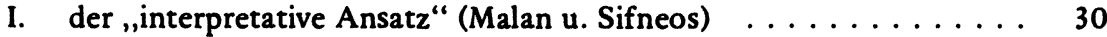

II. der "existentialistische Ansatz" (Mann u. Goldberg) . . . . . . 31

III. der ",korrektive Ansatz" (anlehnend an Alexander u. French) . . . . 34

6. Psychoanalytische Kurztherapie in Forschung und vergleichender

Psychotherapieforschung $\ldots \ldots \ldots \ldots \ldots \ldots \ldots \ldots \ldots \ldots$

7. Kurzzusammenfassung und Uberblick über die Beiträge dieses Buches . . 39 
II. Psychoanalytische Fokaltherapie - Eine klassische Psychoanalytische Kurztherapie in Institutionen

M. Leuzinger-Bobleber

A. Abklärung und Indikationsstellung zur Fokaltherapie . . . . . . . 54

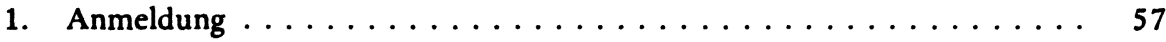

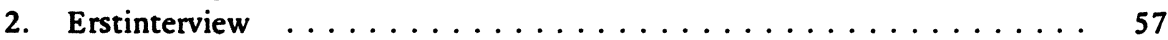

3. Teamgespräch $\ldots \ldots \ldots \ldots \ldots \ldots \ldots \ldots \ldots \ldots \ldots$

4. Psychoanalytisches Zweitinterview ............... 59

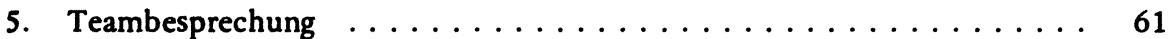

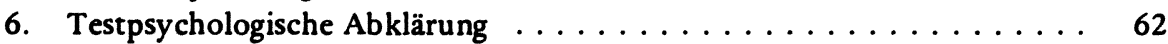

7. Teambesprechung und Fokusbestimmung (Indikationsstellung) . . . 63

8. Drittes Interview und Indikationsbesprechung . . . . . . . . . . . 69

B. Psychoanalytische Fokaltherapie $\ldots \ldots \ldots \ldots \ldots \ldots \ldots \ldots \ldots$

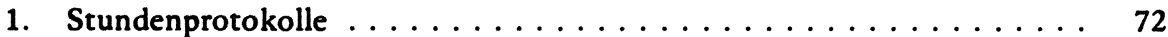

2. Diskussion der Falldarstellung $\ldots \ldots \ldots \ldots \ldots \ldots \ldots$

2.1 Ergänzende Informationen zur Psychodynamik des Patienten . . . . 84

2.2 Einige Überlegungen zur fokaltherapeutischen Behandlungstechnik . 87

a) Eröffnungsphase $\ldots \ldots \ldots \ldots \ldots \ldots \ldots \ldots$

b) Erste Deutungsphase: Fokaler Konflikt in der aktuellen Lebenssituation ....................... 89

c) Erste Durcharbeitungsphase . . . . . . . . . . . . 89

d) Zweite Deutungsphase: Fokaler Konflikt in der Übertragungssituation ...................... 90

e) Zweite Durcharbeitungsphase .................. 90

f) Durcharbeitung der Trennung $\ldots \ldots \ldots \ldots . \ldots \ldots$

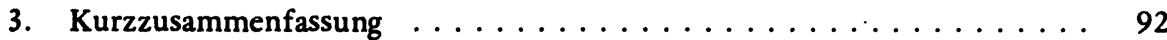

III. Versuch einer Standortbestimmung der Fokaltherapie als einer psychoanalytischen Kurztherapie

R. Klüwer

1. Einführung: Fokaltherapie und Fokalkonferenz als ,psychoanaly tisches Laboratorium" . . . . . . . . . . . . . . . . . . . . . . . . . . 94

2. Die Einstellung des Analytikers $\ldots \ldots \ldots \ldots \ldots \ldots \ldots$

3. Fokaltherapeutische Konzepte .................. 103

3.1 Das szenische Verstehen und die psychoanalytische Methode . . . . 103

3.2 Aktualisierung . . . . . . . . . . . . . . . . . . . . 104

3.3 Struktur und Geschichte . . . . . . . . . . . . . . 106

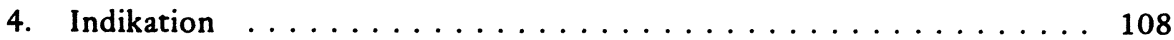

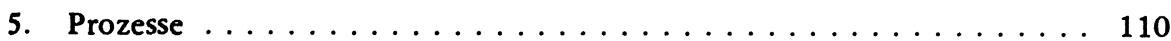

6. Schluß .......................... 111 
IV. Therapiezeit und Lebenszeit: Psychoanalytische Kurzpsychotherapie als Todeserfahrung - Eine kritische Glosse

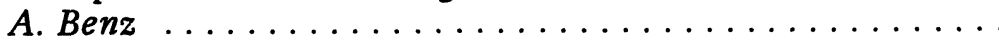

V. Psychoanalytische Kurztherapie in psychiatrischen Settings Ein Beitrag der Psychoanalyse zur Sozialtherapie

A. Gaertner

1. Uber einige Schwierigkeiten psychotherapeutischer Praxis in der Sozialpsychiatrie . ....................... 132

2. Der Ideologieverdacht gegenüber der Psychoanalyse $\ldots \ldots \ldots \ldots \ldots 137$

3. Psychoanalyse in Institutionen $\ldots \ldots \ldots \ldots \ldots \ldots \ldots \ldots \ldots . \ldots \ldots$

4. Erfahrungen mit analytischer Kurztherapie in der Sozialpsychiatrie . . . 145

VI. Zwischen Innen und Außen - Zur angewandten Psychoanalyse in der Psychiatrie

H.-R. Schneider

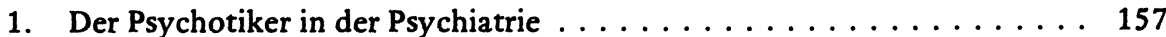

2. Der soziale Ort der Psychiatrie $\ldots \ldots \ldots \ldots \ldots \ldots \ldots \ldots \ldots \ldots 161$

3. Das psychiatrische Team ..................... 164

VII. Angewandte Psychoanalyse in der medizinischen Klinik E. Dreifuss Kattan, I. Honsalek Blaschegg, F. Meerwein

1. Das Funktionsprinzip Liaison-psychiatrischer Tätigkeit an einer medizinischen Universitätsklinik (am Beispiel Zürich) . . . . . . . . . . . . 169

2. Zur Qualifikation des Liaison-Psychiaters, bzw. -Analytikers . . . . . 172

3. Zur Arbeitsweise des Liaison-Analytikers . . . . . . . . . . . . 173

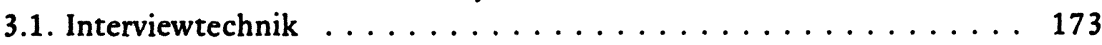

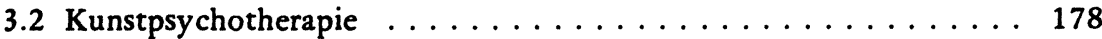

3.3 Arzte und Teamgruppen . . . . . . . . . . . . . . . 180

4. Allgemeine Probleme der Liaison-psychiatrischen Arbeit . . . . . . . . 181

5. Schlußfolgerungen . . . . . . . . . . . . . . . . . . 184

VIII. Die psychoanalytisch orientierte Krisenberatungsstelle R. Büchi, E. Wirth

1. Was ist eine Krise? . . . . . . . . . . . . . . . . 188

2. Die Krisenberatungsstelle am Institut für Angewandte Psychologie . . . 191 
3. Was leistet eine psychoanalytisch orientierte Krisenberatung? . . . . . . 192

3.1 Stützende Elemente . . . . . . . . . . . . . . . . . . 193

3.2 Klären und Strukturieren einer Situation . . . . . . . . . . . 195

3.3 Konfliktwahrnehmung und Einsicht . . . . . . . . . . . 198

3.4 Auseinandersetzung mit dem Konflikt . . . . . . . . . . . . . . 200

4. Institutionelle Einflüsse auf die Möglichkeiten der Krisenberatung . . . . 202

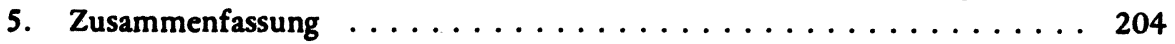

IX. Psychoanalytikerin in einer Child Guidance Clinic

E. Brainin

1. Zur Geschichte der Child Guidance Clinic . . . . . . . . . . . . . 205

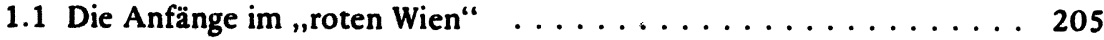

1.2 Die Nachkriegszeit . . . . . . . . . . . . . . . 208

2. Psychoanalytiker in sozialen Einrichtungen . . . . . . . . . . . . 209

3. Die Vorgehensweise am Institut (mit zwei Falldarstellungen) . . . . . . 212

4. Die fürsorgerische Tätigkeit $\ldots \ldots \ldots \ldots \ldots \ldots \ldots \ldots \ldots \ldots$

X. Psychologische Beratungsstelle für Frauen Zürich Eine Auseinandersetzung mit Fragen, die sich momentanen Antworten widersetzen

M. Buess, I. Freye, M. Grüntzig-Seebronner, M. Gubelmann, B. Koch, E. Wandeler-Deck

1. „Frauen beraten Frauen“ - Psychoanalytisches Arbeiten an der Institution Psychologische Beratungsstelle für Frauen . . . . . . . . 225

2. Macht und Arbeit von Frauen im Spannungsfeld von weiblichem Lebenszusammenhang und gesamtgesellschaftlicher Orientierung . . . . . 230

3. Wie arbeiten wir damit? Was bringt uns dieser Ansatz? . . . . . . . . 232

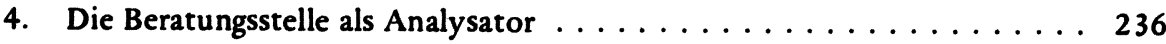

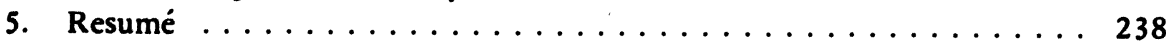

XI. Psychoanalyse und Institution - Eine Bilanz

A. Bauleo

Kommentierte Kurzbibliographie 


\section{Vorwort}

Seit es die Psychoanalytische Kurztherapie gibt, ist sie Gegenstand heftiger Diskussionen und Auseinandersetzungen. Viele Psychoanalytiker betrachten es auch heute noch als eine Paradoxie, einen psychoanalytischen Prozeß zum vornherein zeitlich limitieren zu wollen. Andere hingegen sehen gerade in diesem Setting die Chance, spezielle Themen wie Zeit, Trennung und Tod optimal psychoanalytisch bearbeiten zu können. - Von den einen als Möglichkeit gepriesen, endlich psychoanalytisches wissen und therapeutische Erfahrungen breiteren, weniger privilegierten Bevölkerungskreisen zugänglich zu machen, wird die Psychoanalytische Kruztherapie von anderen angeprangert, sie "verrate" die Substanz der Psychoanalyse und opfere den sie auszeichnenden Erkenntnisanspruch einem "nur" therapeutischen Anliegen.

Diese und andere Widersprüche werden vor allem für jüngere Psychoanalytiker relevant, die versuchen, in Institutionen psychoanalytisch zu arbeiten. In den meisten Institutionen ist es nicht möglich, "große Analysen" (3-5 wöchentliche sitzungen mit dem gleichen Patienten) durchzuführen. I. d. R. schreiben die Rahmenbedingungen zeitlich limitierte Therapieverfahren fest. Diese insititutionelle situation weckt daher oft ein starkes Interesse an allen Formen der Psychoanalytischen Kurztherapie.

In diesem Band berichten verschiedene Teams von Psychoanalytikern über ihre Tätigkeit in Institutionen wie Beratungsstellen, Psychiatrischen und Medizinischen Kliniken, einer Krisenberatungsstelle, einer Child Guidance Clinic und einer Frauenberatungsstelle. Diese Auswahl ist sicher nicht ganz repräsentativ für die Breite des spektrums von Institutionen, in denen Psychoanalytiker arbeiten. Dennoch kann aber ein Eindruck von der vielfalt der Anwendbarkeit von Psychoanalytischen Kurztherapien, ihren Konzepten und den damit verbundenen klinischen und institutionellen Problemen vermittelt werden (detaillierte übersicht über die einzelnen Beiträge: vgl. 7. der Einführung ( $I$ ) ).

Einem weiteren Anliegen können wir im Rahmen dieses Buches nur ungenügend gerecht werden. Wir sind zwar überzeugt, daß die klinisch therapeutische Arbeit wesentlich von der Dimension des institutionellen Settings mitgeprägt wird, in dem sie stattfindet. Doch können wir in den meisten Beiträgen dieser Einflußquelle nicht im Sinne einer stringenten Institutionsanalyse (vgl. AUTHIER \& HESS, 1981; LAPASSADE 1972; LOUREAU 1970 u. a.) nachgehen, sondern müssen uns - der vorwiegend klinischen zielsetzung unseres 
Buches entsprechend - darauf beschränken, von unserem subjektiven Erleben und unseren Erfahrungen dieser institutionellen Seite unserer Tätigkeit zu berichten. Einzig A. BAULEO widmet sich ausschließlich dem Thema: "Psychoanalyse und Institution", doch kann seine knappe Zusammenfassung kaum mehr als ein Hinweis auf einige Hauptaspekte dieser Problematik sein und auf weitere Lektüre verweisen.

Dieses Buch wäre nicht entstanden ohne die spontane zusage zur Mitarbeit vieler meiner psychoanalytischen Kolleginnen und Kollegen, den Mitautoren dieses Bandes. Ich danke ihnen für die fruchtbare gedankliche und praktische Zusammenarbeit. Regula Schiess verdanke ich den näheren Kontakt zu Armando Bauleo und Madeleine Dreyfus, daß die Aufnahme seines Beitrages in das Buch nicht noch im letzten Moment an übersetzungsschwierigkeiten scheiterte. Horst Kächele danke ich für die genaue und konfrontierende Lektüre der "Einführung" und Brigitte Borke für die graphische Gestaltung der Abbildungen. Herr Dr. Martin Weinmann vom Westdeutschen Verlag ermöglichte durch seine fachmännische und unkompliziert freundliche $\mathrm{Hilfe}$ eine neue Konzeption der geplanten Publikation, die u. a. durch das Entgegenkommen und die rasche und zuverläßige Texterfassung und -gestaltung durch Katrin und Hartmut Lamott (HEUREKA Computer, Glattbrugg-Zürich) relativ preiswert erscheinen kann.

Meine persönliche Motivation zur Auseinandersetzung mit dem Thema dieses Buches ist vor allem verbunden mit den Erfahrungen während meiner Assistentenzeit an der Beratungsstelle der Abteilung für Klinische Psychologie der Universität Zürich. Ich danke meinen Kollegen Michaela Grüntzig und Rainer Krause, unseren kritischen studenten sowie dem Institutsleiter, Prof. Ulrich Moser für die zwar anstrengende, aber auch produktive zeit an dieser Institution.

Werner Bohleber danke ich für die vielen Diskussionen, das Mitdenken und das kritische Lesen meiner Manuskripte. 